Bull. Austral. Math. Soc.

Vol. 40 (1989) [97-108]

\title{
DEGENERATE CRITICAL POINTS, HOMOTOPY INDICES AND MORSE INEQUALITIES III
}

\author{
E.N. DANCER
}

\begin{abstract}
This paper contains two main types of results. Firstly, we discuss what sort of critical points are obtained in various direct and dual minimax principles. The techniques we apply are widely applicable. Secondly, we obtain "best possible" results on which critical points of a function on $R^{n}$ are removable.
\end{abstract}

\section{INTRODUCTION}

This paper is a continuation of $[6]$. We prove three main results. We improve the result in $[\boldsymbol{\theta}]$ on what cort of critical point is obtained in the saddle point theorem. This was motivated by seeing an announcement of the results of [13] for non-degenerate critical points. In addition, we prove similar results for a number of dual variational principles. Indeed the proofs in this case are much simpler than the proofs in $[\boldsymbol{6}]$ and the methods seem easy to apply in other situations. In particular, the technicalities associated with the fundamental group in $[\boldsymbol{\theta}]$ do not appear. Solinini and Lazer [13] have independently obtained results related to these for the case of non-degenerate critical points. For this case, our results agree with theirs or are better. The results which they obtain for degenerate critical points are much weaker than ours. (On the other hand, they do consider some other different minimax points.) We also considerably improve the result in [6] on the removability of critical points. We now consider our results in more detail.

Firstly, consider Rabinowitz's saddle point theorem [18]. If we look at the critical value $c^{\prime}$ obtained by a "dual" minimal agrument, we prove that there is a critical point $\bar{x}$ in $f^{-1}\left(c^{\prime}\right)$ such that the Betti number $B^{i}(f, \bar{x}) \neq 0$ for some $i \geqslant \operatorname{dim} W$ where $W$ is the subspace in the saddle point theorem on which $f$ is bounded below. In particular, if $W$ is one or two-dimensional, we can take $i=\operatorname{dim} W$. In addition if $\operatorname{dim} W>1$, we show that for the most common critical level $c$ occuring in the saddle point theorem (the one used in [6]), there is a critical point $\bar{x}$ in $f^{-1}(c)$ such that $B^{i}(f, \bar{x}) \neq 0$ for some $i$ with $2 \leqslant i \leqslant \operatorname{dim} W$. This improves Theorem 3 in [6] by replacing " $1 \leqslant i \leqslant \operatorname{dim} W$ "

Received 30 September 1988

1 should like to thank A. Lazer for sending me a preprint of [13] prior to publication and the Centre for Mathematical Analysis in Canberra for hospitality. Much of this paper was written on a visit there.

Copyright Clearance Centre, Inc. Serial-fee code: 0004-9729/89 \$A2.00+0.00. 
by " $2 \leqslant i \leqslant \operatorname{dim} W$ ". In both cases, we obtain better results in the infinite-dimensional case than in [13].

Secondly, we prove the conjecture in [6] on sufficient conditions for a critical point on $R^{n}$ (where $n \geqslant 6$ ) to be removable. (A critical point $\bar{x}$ of $f$ is said to be removable if we can find a neighbourhood $U$ of $\bar{x}$ and $C^{2}$ functional $g$ such that $g=f$ outside $U$ but $g$ has no critical points in $\bar{U}$.) Our condition is useful because is easy to verify in many cases. We also indicate how our methods can be used to obtain a rather more complicated necessary and sufficient condition for removability if $n \geqslant 6$.

The techniques in Sections $1-2$ here and in [6] are general techniques which can be used to study many other variational problems where there are degenerate critical points. It seems to the author that the direct treatment of degenerate critical points, while it is technical, is always likely to give better results than approximation arguments. Note that a major motivation for studying what sort of critical points we obtain in minimax problems is that such results can often be used to prove the existence of additional critical points (as in [5], Section 4).

In Section 1, we prove the result on the type of critical points obtained in several dual variational principles. In Section 2, we improve the result in [6] on the type of critical point obtained in the most usual critical level in the saddle point theorem. We also consider infinite-dimensional generalisations. Funally, in Section 3, we discuss the removability of critical points.

Our notation follows that in [5] and [6], and a copy of [6] would be helpful for reading Section 2.

\section{The Type of CRitical points in dUal Variational pRinciples}

In this section, we obtain information on the cohomology of critical points in some dual variational principles. We prove a result in detail in one case and sketch the proof of another result. However, the techniques can be used for a number of other problems.

Assume that $f: \boldsymbol{R}^{n} \rightarrow \mathbf{R}$ is $C^{2}$ and that there is a subspace $W$ of $R^{n}$ and an $r>0$ such that

$$
b=\max \{f(x): x \in W,\|x\|=r\}<\inf \left\{f(x): x \in W^{\perp}\right\}
$$

and $f$ satisfies the Palais-Smale condition. Let $D_{r}=\{x \in W:\|x\| \leqslant r\}$.

Let $\mathcal{A}$ denote the class of compact subsets $A$ of $E$ with property that for every continuous map $\sigma: A \rightarrow W$ such that $\sigma \mid A \cap \partial D_{r}=I d, \sigma$ has a zero in $A$. If $h: E \rightarrow E$ is a homeomorphism such that $\left.h\right|_{\partial D_{r}}=I d$, one easily sees that $h(A) \in \mathcal{A}$ is $A \in \mathcal{A}$.

Then one easily shows that

$$
c^{\prime}=\inf _{A \in \mathcal{A}} \sup _{x \in A} f(x)
$$


is a critical value of $f$ and $c^{\prime}>b$. See [13], though similar results appeared elsewhere earlier. (See Solimini [20].)

We need some notation from [5]. Assume that zero is a critical point. Let $\pi(t, x)$ denote the flow corresponding to the differential equation $x^{\prime}(t)=-\nabla f(x(t))$, let $E=$ $\left\{x \in R^{n}: \pi(t, x) \rightarrow 0\right.$ as $\left.t \rightarrow-\infty\right\}$ and let $E_{\varepsilon}=E \cap f^{-1}(-\varepsilon)$. E is known as the entry set (for 0 ). It is shown in [5, p.6] that $E_{\varepsilon}$ is close to zero if $\varepsilon$ is small. Choose a manifold neighbourhood $N_{e}$ of $E_{e}$ in $f^{-1}(-\varepsilon)$ and let

$$
U_{\varepsilon}=\{0\} \cup\left\{\pi(t, x): t \leqslant 0, x \in N_{\varepsilon}, f(\pi(t, x)) \leqslant \varepsilon\right\} \cup \tilde{I}_{\varepsilon}
$$

where $\tilde{I}_{s}=\bigcup_{0 \leqslant t \leqslant s} I_{t}$ and $I_{s}=\{x: \pi(t, x) \rightarrow 0$ as $t \rightarrow \infty, f(x)=s\}$. Thus, $U_{\varepsilon}$ is constructed from $N_{e}$ by following the flow up to $f^{-1}(\varepsilon)$ and adding points $x$ such that the flow tends to zero as $t \rightarrow \infty$. If $\varepsilon$ is small, it is shown on p.7 of [5] that $U_{e}$ is a small neighbourhood of zero, $h(-\nabla f, 0)=\left[U_{\epsilon} / N_{\epsilon}\right]$ and $U_{\varepsilon}$ can be chosen connected. We can use a similar construction near any critical point $\bar{x} . U_{e}$ will then be a subset of $f^{-1}[c-\varepsilon, c+\varepsilon]$ where $c=f(\bar{x}) . \tilde{H}^{j}(f, \bar{x}, G)$ denotes the local cohomology of $f$ at $\bar{x}$ with coefficients in $G$ and $B^{j}(f, \bar{x}, G)$ the corresponding Betti numbers (as in [6]). Also, $f^{a}$ is used as an abbreviation for $f^{-1}((-\infty, a])$.

TheOREM 1. Under the above assumptions and if each critical point in $f^{-1}\left(c^{\prime}\right)$ is isolated in $\mathbf{R}^{n}$ then there exists a critical point $\bar{x}$ in $f^{-1}\left(c^{\prime}\right)$ such that $\widetilde{H}^{j+1}\left(f, \bar{x}, \pi_{j}\left(S^{k-1}\right)\right) \neq\{0\}$ for some $j \geqslant k-1$ where $k=\operatorname{dim} W$. If $k=1$ or 2 , we can take $j=k-1$. Thus $B^{j}\left(f, \bar{x}, Z_{p}\right) \neq 0$ for some $j \geqslant k$ and for some prime $p$ while if $k=1$ or 2 we can take $j=k$.

Proof: Suppose $\varepsilon>0$ and choose box-like neighbourhood $U_{\varepsilon}^{i}$ of the critical points $\left\{x_{i}\right\}_{i=1}^{m}$ in $f^{-1}\left(c^{\prime}\right)$ such that the $U_{e}^{i}$ are disjoint and, as usual, $U_{e}^{i}$ intersects $f^{c^{\prime}-\epsilon}$ in $N_{c}^{i}$. By the deformation lemma (see $[18, p .162]$ ) and by our earlier remark on the invariance of $\mathcal{A}$ under suitable homeomorphisms, we see that there exists $A \in \mathcal{A}$ such that $f(x) \leqslant c^{\prime}+\varepsilon$ on $A$ and $A \subseteq f^{c^{\prime}-\varepsilon} \cup \bigcup_{i=1}^{m} U_{\varepsilon}^{i}$. (Remember that, in the deformation lemma, we can obtain a homeomorphism which fixes $f^{c^{\prime}-\varepsilon}$ and with range in $\left.f^{c^{\prime}-\varepsilon} \cup \bigcup_{i=1}^{m} U_{e}^{i}\right)$. If $A \in \mathcal{A}, B$ is compact and $A \subseteq B$, then $B \in \mathcal{A}$. Thus we can assume without loss of generality that $U_{\varepsilon}^{i} \subseteq A$ for $i=1, \ldots, m$. Now $A \backslash$ $\bigcup_{i=1}^{m}\left(U_{e}^{i} \backslash N_{e}^{i}\right) \notin \mathcal{A}$ since otherwise it would follow that we would have an element of $\mathcal{A}$ which is contained in $f^{c^{\prime}-e}$. This would contradict the definition of $c^{\prime}$. Since $G=A \backslash \bigcup_{i=1}^{m}\left(U_{e}^{i} \backslash N_{e}^{i}\right) \notin \mathcal{A}$, there is a continuous function $\varphi: G \rightarrow W$ such that $\varphi(x)=x$ on $G \cap \partial D_{r}$ but $\varphi(x) \neq 0$ on $G$. Since $c^{\prime}>b$, our construction ensures that 
$U_{e}^{i} \cap \partial D_{r}=\emptyset$ for $1 \leqslant i \leqslant m$. Now there must be an $i$ such that $\left.\varphi\right|_{N_{\varepsilon}^{i}}$ has no continuous extension to a map of $U_{e}^{i}$ into $W \backslash\{0\}$. Otherwise, we could obtain a continuous map of $G \cup \bigcup_{i=1}^{m} U_{\varepsilon}^{i}=A$ into $W \backslash\{0\}$ such that $\varphi(x)=x$ on $A \cap \partial D_{r}$. This contradicts our assumption that $A \in \mathcal{A}$. Now $W \backslash\{0\}$ has the same homotopy type as $\partial D_{r}$ and thus of $S^{k-1}$. Hence we see that there is an $i$ and a continuous map $\widetilde{\varphi}: N_{\varepsilon}^{i} \rightarrow S^{k-1}$ such that $\widetilde{\varphi}$ has no continuous extension to a map of $U_{\varepsilon}^{i}$ into $S^{k-1}$. By obstruction theory (see Hu [8, Chapter VI], this implies that $\widetilde{H}^{j+1}\left(U_{e}^{i}, N_{e}^{i}, \pi_{j}\left(S^{k-1}\right)\right) \neq\{0\}$ for some $j$ (Here we are assuming that $k>2$ to ensure that $S^{k-1}$ is simply connected. We return to the other cases in a moment.) Since $\pi_{j}\left(S^{k-1}\right)=\{0\}$ if $j<k-1$ and since

$$
\widetilde{H}^{j+1}\left(f, x_{i}, \pi_{j}\left(S^{k-1}\right)\right)=\widetilde{H}^{j+1}\left(U_{e}^{i}, N_{e}^{i}, \pi_{j}\left(S^{k-1}\right)\right)
$$

(see $[5, \mathrm{p} .3]$ ) the result follows. If $k=2$, we can use $\mathrm{Hu}$ [8, Corollary II.7.4] to show that the only obstruction lies in $\widetilde{H}^{2}\left(U_{e}^{i}, N_{e}^{i}, Z\right)$ and we can complete the proof as before. If $k=1, S^{k-1}$ is a two point space and the only way an extension can fail to exist is if two distinct components of $N_{\varepsilon}^{i}$ lie in the same component of $U_{\varepsilon}^{i}$. It follows that $\widetilde{H}^{1}\left(f, x_{i}, z\right) \neq\{0\}$. (Otherwise, as we showed in [5, p.7 and p.10], we can choose $U_{\varepsilon}^{i}$ and $N_{e}^{i}$ both connected.) The last statement follows from the universal coefficient theorem $[\mathbf{2 1}, \mathrm{p} .246]$. This completes the proof.

Remarks. As usual, we can improve the result if $k=1$ and either $f^{\prime \prime}(x)$ has a negative eigenvalue or $\operatorname{dim} N\left(f^{\prime \prime}(x)\right) \leqslant 1$, for every critical point $x$ in $f^{-1}\left(c^{\prime}\right)$. In this case, as in $[\mathbf{5}, \mathbf{p . 1 0}]$, we can deduce that

$$
\widetilde{H}^{i}(f, \bar{x}, Z)= \begin{cases}Z & \text { if } i=1 \\ \{0\} & \text { otherwise }\end{cases}
$$

If $k=2$, our result says that $\widetilde{H}^{2}(f, \bar{x}, Z) \neq\{0\}$. In particular, if $\bar{x}$ is non-degenerate, $f^{\prime \prime}(\bar{x})$ has exactly two negative eigenvalues. This improves Theorem 2.2 in [13] in the finite-dimensional case. Note also that, in the finite-dimensional case, Theorem 1 improves Theorems 2.2 and 2.5 in [13].

The same idea can be used to obtain results for a number of other dual variational principles. For example, assume that $f: \mathbf{R}^{n} \rightarrow \mathbf{R}$ is $C^{2}, f(0)=0, f$ is even and satisfies the Palais-Smale condition. Assume that $k \geqslant 1$ and

$$
-\infty<C_{k+1}=\inf _{A \in \Gamma_{k+1}} \sup _{x \in A} f(x)<0
$$

where $\Gamma_{m}$ denotes the compact, symmetric subsets of $\mathbf{R}^{n}$ which do not contain zero and which have genus at least $m$. Here a compact symmetric set $A$ not containing 
zero has genus greater than or equal to $t$ if and only if there is no continuous odd map $\varphi: A \rightarrow R^{e} \backslash\{0\}$ for $e<t$ (see Rabinowitz [19]). Then $C_{k+1}$ is a critical value of $f$ (see [3]). If there are only a finite number of critical points on $R^{n}$ then the conclusion of Theorem 1 holds. (Note also that, if $k=0$, we would have a local minimum of $f$ ). We merely sketch this. If $\varepsilon>0$, we can choose $A \in \Gamma_{k+1}$ such that $A \subseteq f^{C_{k+1}-\varepsilon} \cup \bigcup_{i=1}^{m} U_{\varepsilon}^{i}$ where $U_{\varepsilon}^{i}$ are our usual box-like neighbourhoods of the critical points. We can choose the $U_{e}^{i}$ so as to preserve the symmetry and, as in the proof of Theorem 1, we can ensure that $U_{\varepsilon}^{i} \subseteq A$. Since $G=A \backslash \bigcup_{i=1}^{m}\left(U_{\varepsilon}^{i} \backslash N_{\varepsilon}^{i}\right)$ is not in $\Gamma_{k+1}$, genus $G<k+1$. Hence there is a continuous odd map $\psi: G \rightarrow \mathbf{R}^{k} \backslash\{0\}$. However, since genus $A \geqslant k+1, \psi$ does not extend to a continuous odd map of $A$ into $\mathrm{R}^{k} \backslash\{0\}$. This implies that for some $i,\left.\psi\right|_{N_{\varepsilon}^{i}}$ does not extend to a continuous map of $U_{\varepsilon}^{i}$ into $R^{k} \backslash\{0\}$. (We can preserve the oddness by noting that, if we can define $\psi$ on $U_{e}^{i}$ we can define $\psi$ on $U_{e}^{j}$ (where $-x_{i}$ lies in $U_{\varepsilon}^{j}$ ) by using the oddness.) The proof can now be completed in the same way as in Theorem 1. A related but weaker result appears in [1].

Remarks. In this case, we obtain good results for $k=0,1,2$.

\section{AN IMPROVEMENT ON OUR EARLIER RESULT ON THE SADDLE POINT THEOREM}

In this section, we improve our result in [6] on the type of critical point obtained in the saddle point theorem.

As in Section 1, assume that $f: \mathbf{R}^{n} \rightarrow \mathbf{R}$ is $C^{2}$ and that there is a subspace $W$ of $\mathrm{R}^{n}$ and an $r>0$ such that

$$
b=\max \{f(x): x \in W,\|x\|=r\}<\inf \left\{f(x): x \in W^{\perp}\right\}
$$

and $f$ satisfies the Palais-Smale condition.

Let $\Gamma$ denote the set of continuous maps $\gamma: D_{r} \rightarrow H$ such that $\left.\gamma\right|_{\partial D_{r}}=I d$. Rabinowitz [18, Theorem 1.2] proved that, under these assumptions,

$$
c \equiv \inf _{\gamma \in \Gamma} \sup _{x \in D_{r}} f(\gamma(x))
$$

is a critical value of $f$ and $c>b$. Let $f^{a}=\left\{x \in \mathbb{R}^{n}: f(x) \leqslant a\right\}$.

We say that $c$ is a nice critical value of $f$ if, for every critical point $\bar{x}$ in $f^{-1}(c)$ (i) $\operatorname{dim} N\left(f^{\prime \prime}(\bar{x})\right) \leqslant 2$ or (ii) $f^{\prime \prime}(\bar{x})$ has at least one negative eigenvalue or (iii) $\bar{x}$ is a local minimim of $f$.

THEOREM 2. If the above assumptions hold, if each critical point in $f^{-1}(c)$ is isolated in $\mathbf{R}^{n}$, if $c$ is a nice critical value of $f$ and if $k \geqslant 2$, then there is a critical point $\bar{x}$ in $f^{-1}(c)$ such that $\widetilde{H}_{j}(f, \bar{x}, Z) \neq\{0\}$ for some $j$ in $\{2, \ldots, k\}$. 
Remarks. This improves Theorem 3 in $[6]$ by replacing $j \in\{1, \ldots, k\}$ by $j \in$ $\{2, \ldots, k\}$.

Proof: Let $\left\{x_{i}\right\}_{i=1}^{s}$ denote critical points in $f^{-1}(c)$ and, if $\varepsilon$ is small and positive, choose disjoint box-like neighbourhoods $U_{e}^{i}$ of the critical points in $f^{-1}(c)$ as in the proof of Theorem 1. Let $N_{\varepsilon}^{i}=U_{\varepsilon}^{i} \cap f^{-1}(c-\varepsilon)$. As in the proof of Lemma 1 in $[6]$, there is a continuous map $\gamma: D_{r} \rightarrow R^{n}$ such that $\left.\gamma\right|_{\theta D_{r}}$ is the identity and $\gamma(x) \in f^{c-\varepsilon} \cup \bigcup_{i=1}^{j} U_{e}^{i}$ for $x \in D_{r}$. We order the $x_{i}$ such that $\widetilde{H}_{j}\left(f, x_{i}, Z\right)=\{0\}$ if $j>k$ and $i \geqslant m$ (where $m \leqslant s$ ). We next show $\gamma$ can be chosen such that $\gamma(x) \in f^{c-\varepsilon} \cup \bigcup_{i=1}^{m} U_{\varepsilon}^{i}$ for $x \in D_{r}$. This is effectively proved in [6]. In the proof of Lemma 1 there, it is shown that if $\pi_{j}\left(U_{e}^{i}, N_{e}^{i}\right)=\{0\}$ for $1 \leqslant j \leqslant k$, we can modify $\gamma$ on $\gamma^{-1}\left(U_{e}^{i}\right)$ so that it only takes values in $N_{\varepsilon}^{i} \subseteq f^{c-e}$. Now the proof of Theorem 3 in [6] shows that $\pi_{j}\left(U_{\varepsilon}^{i}, N_{e}^{i}\right)=\{0\}$ for $1 \leqslant j \leqslant k$ if and only if $\tilde{H}_{j}\left(U_{\varepsilon}^{i}, N_{\varepsilon}^{i}, Z\right)=\{0\}$ for $1 \leqslant j \leqslant k$. (It is here that we use that $c$ is a nice critical value.) Since $\widetilde{H}_{j}\left(f, x_{i}, Z\right)=\widetilde{H}_{j}\left(U_{\varepsilon}^{i}, N_{\varepsilon}^{i}, Z\right)$, our claim follows. By a similar, but much easier argument we can change $\gamma$ so as not to take values near $x_{i}$ if $x_{i}$ is a local minimum of $f$.

Hence we can choose $\gamma$ such that $\gamma(x) \in f^{c-\varepsilon} \cup \bigcup_{i=1}^{m} U_{\varepsilon}^{i}$ for $x \in D_{r}$ where $\widetilde{H}_{j}\left(f, x_{i}, Z\right) \neq\{0\}$ for some $j$ in $\{1, \ldots, k\}$ (depending on $i$ ) for each $i$ in $\{1, \ldots, m\}$. If our result is false, $\widetilde{H}_{j}\left(f, x_{i}, Z\right)=\{0\}$ for $2 \leqslant j \leqslant k$ and $1 \leqslant i \leqslant m$. Hence $\widetilde{H}_{1}\left(f, x_{i}, Z\right) \neq\{0\}$ for $1 \leqslant i \leqslant m$.

Since $k>1, \partial D_{r}$ is connected and hence $\gamma\left(\partial D_{r}\right)$ lies in a single component $C^{1}$ of $f^{c-\varepsilon}$. Let $M=\bigcup_{i=1}^{m} N_{\varepsilon}^{i} \cap C^{1}$. We set $\widetilde{W}=\left\{x \in D_{r}: \gamma(x) \notin C^{1}\right\}$ and $S=\left\{x \in D_{r}: \gamma(x) \in M\right\}$. Then $S$ is a closed set and $\gamma(S) \subseteq M$.

If we show that the inclusion $\tilde{i}: M \rightarrow f^{c-e}$ is a homotopic to a constant, then it will follow that $\gamma: S \rightarrow f^{c-e}$ is homotopic to a constant (since $\gamma(S) \subseteq M$ ). Thus, by the homotopy extension property, $\gamma$ extends to a map of $\widetilde{W}$ into $f^{c-\varepsilon}$ and hence the result follows. (Note that, if $\varepsilon$ is small, $c-\varepsilon>b$ and thus $\widetilde{W}$ does not intersect $\partial D_{r}$.)

It remains to prove that $\widetilde{i}$ is homotopic to a constant. We first prove that $N_{e}^{i}, i=$ $1, \ldots, m$, can be chosen such that each component is contractible. Consider $x_{1}$. The other cases are similar. Let $E_{1}$ denote the exit set at $x_{1}$ for the flow corresponding to $x^{\prime}=\nabla f(x)$ and let $E_{e}^{1}=E_{1} \cap f^{c-\varepsilon}$. Since $\widetilde{H}_{1}\left(f, x_{1}, Z\right) \neq\{0\}$ the shifting lemma (see [5, Theorem 3]) implies that $f^{\prime \prime}\left(x_{1}\right)$ has zero or one negative eigenvalue. In the latter case, Theorem 4 in [5] implies that 0 must be a local minimum for the corresponding bifurcation equation and hence the exit set for the flow corresponding to the bifurcation equation is empty. We can then use Palmer's change of coordinates as in the proof of Lemma 2 in [6] to calculate the exit set for the flow. By a similar but easier argument 
to that in the proof of Lemma 2 in [6] we see that $E_{c}^{1}$ consists of 2 points. We can then choose $N_{e}^{i}$ to be the union of contractible neighbourhoods of each of these points in $f^{-1}(c-\varepsilon)$. Now consider the case where $f^{\prime \prime}\left(x_{1}\right)$ has no negative eigenvalues. In this case, by the proof of Lemma 2 in [6]. $E_{\varepsilon}^{1}$ is homeomorphic to the corresponding exit set $\widetilde{E}_{\epsilon}$ for the bifurcation equation on $N\left(f^{\prime \prime}\left(x_{1}\right)\right)$ (intersected with $f^{-1}(c-\varepsilon)$ ). We consider the case where $N\left(f^{\prime \prime}\left(x_{1}\right)\right)$ is 2-dimensional. The other cases are easier. Then $\tilde{E}_{e}$ is a closed subset of the one-manifold $f^{-1}(c-\varepsilon)$ in $N\left(f^{\prime \prime}\left(x_{1}\right)\right)$. By [6, p.12], it has only a finite number of components. Hence $\widetilde{E}_{\varepsilon}$ is a finite disjoint union of sets homeomorphic to $[0,1]$. Here we are using essentially that $f^{-1}(c-\varepsilon) \cap N\left(f^{\prime \prime}\left(x_{1}\right)\right)$ is a one-manifold. Hence there is a finite union of disjoint contractible sets in $f^{-1}(c-\varepsilon) \cap$ $N\left(f^{\prime \prime}\left(x_{1}\right)\right)$ with $\widetilde{E}_{\varepsilon}$ in the union of their interiors. It is now easy but tedious to construct nice contractible neighbourhoods of each component of $E_{\varepsilon}^{1}$ in $f^{-1}(c-\varepsilon)$. (One way is to use Palmer's change of co-ordinates and the neighbourhoods of $\widetilde{E}_{e}$ above to construct a neighbourhood in $\tilde{f}^{-1}(c-\varepsilon)$ for a different but related function $\tilde{f}$ and then use the flow to obtain a neighbourhood in $f^{-1}(c-\varepsilon)$. Here $\tilde{f}(x)=1 / 2 f^{\prime \prime}(0) x^{2}+g(P x)$ where $P$ is the orthogonal projection onto $N\left(f^{\prime \prime}(0)\right)$ and the bifurcation equation is the gradient of $g$ on $N\left(f^{\prime \prime}(0)\right)$.

By the previous paragraph, we can obtain contractible neighbourhoods of each component of $E_{\varepsilon}^{1}$. The union of these is defined to be $N_{\epsilon}^{1}$. As in [5], we can then construct the corresponding $U_{e}^{1}$. We do this for each $x_{i}, i=1, \ldots, m$. Let $\left\{A_{j}\right\}_{j=1}^{p}$ be the components which lie in $C_{1}$. Choose $x_{j} \in A_{j}$.

Since each $A_{j}$ is contractible, $\tilde{i}: M \rightarrow f^{c-\varepsilon}$ is homotopic to the map which sends all of $A_{j}$ to $x_{j}$. Since each $x_{j}$ is in the path connected set $C_{1}$, it follows easily that $\tilde{i}$ is homotopic to the map which sends $M$ to $x_{1}$ and hence the result follows.

Remarks. If $k=2$, we have that $\widetilde{H}^{2}(f, \bar{x}, Z) \neq\{0\}$. Our result improves Theorem 3 in [6] and Theorems 2.4 and 2.7 in [13] (at least in the finite-dimensional case).

With more care, we can replace $\operatorname{dim} N\left(f^{\prime \prime}(\bar{x})\right) \leqslant 2$ by $\operatorname{dim} N\left(f^{\prime \prime}(\bar{x})\right) \leqslant 3$ in the definition of a nice critical point and retain Theorem 2. This depends upon results in [11]. As in [6], we can use the universal coefficient theorem to deduce that $B^{j}\left(f, \bar{x}, Z_{p}\right) \neq 0$ for some prime $p$ and some $j$ in $\{2, \ldots, k\}$. Remember that $\widetilde{H}_{1}(f, \bar{x}, Z)$ and $\widetilde{H}^{1}(f, \bar{x}, Z)$ have no torsion.

It is unclear if our techniques can be used to obtain a result for the critical value $\bar{c}$ of $f$ in [13]. We could certainly use our ideas to give an alternative proof of Theorems 2.3 and 2.65 in [13]. In the case of degenerate zeros, one might hope to prove that there is a critical point $\bar{x}$ in $f^{-1}(\bar{c})$ such that $B^{i}\left(f, \bar{x}, Z_{p}\right) \neq 0$ for some $i$ in $\{1, \ldots, k\}$ and some $p$ and for some $i$ in $\{k, k+1, \ldots\}$ and some $p$ (where the two $p$ 's may be different). This appears rather harder to prove. 
As one might expect, one can prove the natural analogues of Theorems 1 and 2 for maps $f$ on a Hilbert space which satisfy the Palais-Smale condition and where $D^{2} f(\vec{x})$ is Fredholm at each critical point. This is in line with the author's contention that if one can solve a problem in the large finite dimensional case, it is often easy to modify the proof to do the infinite-dimensional problem. We show briefly how this can be done We need to use the definition of the homology of a critical point in [7]. We first consider Theorem 9 in [6] in the infinite-dimensional case. By Mawhin and Willem [16], we can, by using a local homeomorphism, assume that near a critical point $\bar{x}, f(\bar{x}+x)=f(\bar{x})+1 / 2\left\|P_{1} x\right\|^{2}-1 / 2\left\|P_{2} x\right\|^{2}+g\left(P_{0} x\right)$ where $P_{1}\left(P_{2}\right)$ is the orthogonal projection onto the space spanned by the eigenvectors corresponding to positive (negative) eigenvalues, $P_{0}=I-P_{1}-P_{2}, g(0)=g^{\prime}(0)=0$. Note that, by the definition of the homology of a critical point in [7], it is not changed by a local homeomorphism. Since decreasing $\left\|P_{1} x\right\|^{2}$ decreases $f$ near $\bar{x}$, we see that we can also choose the function $\gamma$ in the definition of $c$ such that, if $\gamma(x)$ is near $\bar{x}$, then $\gamma(x)$ is in $\left\{\bar{x}+x: P_{1} x=0\right\}$. Note that, by the shifting lemma, the Betti numbers on this subspace are the same as on the whole space. We can now choose our good pair $(V, \tilde{V})$ as in the proof of Lemma 1 in $[6]$ on the subspace $\left\{x: P_{1} x=0\right\}$ where for simplicity we are assuming that $\bar{x}=0$. The proof of Lemma 1 still shows that $\pi_{j}(V, \widetilde{V}) \neq\{0\}$ for some $j$ in $\{1, \ldots, k\}$. For the moment, let us assume that $R\left(P_{2}\right)$ is finite-dimensional. In this case one obtains the result essentially as in the proof of Lemma 2 and Theorem 3 in [0] except one uses a different flow. One uses the flow generated by the gradient of $-1 / 2\left\|P_{2} x\right\|^{2}+g\left(P_{0} x\right)$ in the subspace of $\left\{x: P_{1} x=0\right\}$. Since this space is a finite-dimensional space we can use the homotopy index methods in $[\boldsymbol{6}]$ and obtain the result. The exit sets will be different from those in $[\theta]$ because we are using a different flow. (Note that this means we can avoid using Palmer's theorem). Now assume $R\left(P_{2}\right)$ is infinite-dimensional. Let us first assume that $\bar{x}$ (where without loss of generality $\bar{x}=0)$ is non-degenerate. In this case we can take our pair $(V, \widetilde{V})$ to be a ball $B$ and its boundary $\partial B$ in an infinite-dimensional Hilbert space. Since $B$ and $\partial B$ are contractible in an infinite-dimensional Banach space (see Bessaga and Pelczynski $[\mathbf{2}$, Corollary III.5.1]) $\pi_{j}(B, \partial B)=\{0\}$ for $j=1, \ldots, k$. This contradicts Lemma 1 in [6]. Now suppose that $\bar{x}=0$ is degenerate but $R\left(P_{2}\right)$ is infinite-dinensional. In this case one can use a standard approximation lemma (see Marino and Prodi [15, Lemma 1.2]) to modify $f$ near 0 to $\widetilde{f}$ so that $\tilde{f}$ has only non-degenerate critical points near 0 , each is at the same level ${ }^{\circ} f(0)$ and each has $R\left(P_{2}\right)$ infinite-dimensional. We can now combine our argument above with the proof of Lemma 1 in $[\mathbf{6}]$ to deduce that we can modify $\gamma$ so that $\gamma$ does not take values in the $U_{e}$ 's for each of the critical points near 0. In particular $\widetilde{f}(\gamma(x))<f(0)$ when $\gamma(x)$ is small. We can now deform along gradient lines (that is, using the deformation lemma) to obtain a new $\gamma$ such that $\gamma(x)$ 
is never close to zero. Thus $\tilde{f}(\gamma(x))=f(\gamma(x))$ for all $x$ in $D_{r}$ (since $f$ and $\tilde{f}$ only disagree near zero). Thus, if zero is a critical point with $R\left(P_{2}\right)$ infinite-dimensional we can always choose $\gamma$ so that $\gamma(x)$ is never close to zero. Thus the critical point with $\pi_{j}(V, \vec{V}) \neq\{0\}$ for some $j$ in $\{1, \ldots, k\}$ obtained in the proof of Lemma 1 in [6] must have $R\left(P_{2}\right)$ finite-dimensional. This completes the proof of the infinitedimensional analogue of Theorem 3 in [6]. The same ideas can be used to prove an infinite-dimensional version of Theorem 2 here. One combines the proof of Theorem 2 here with the ideas above. Once again one chooses $\gamma$ such that, near a critical point $\bar{x}, \gamma$ takes values in $\left\{\bar{x}+x: P_{1} x=0\right\}$, one chooses $U_{\varepsilon}, N_{\varepsilon}$ for the gradient flow of $-1 / 2\left\|P_{2} x\right\|^{2}+g\left(P_{0} x\right)$ on $\left\{\bar{x}+x: P_{1} x=0\right\}$ and one uses this flow rather than the flow for the original equation. Otherwise the proof is much as before.

One can also prove a version of Theorem 1 here in the infinite-dimensional case. We sketch a proof of this. One shows that $A \in \mathcal{A}$ can be chosen such that, near a critical point $\bar{x}, A \subseteq\left\{\bar{x}+x: P_{1} x=0\right\}$. First suppose that $R\left(P_{2}\right)$ is always finite-dimensional. To simplify the notation, we assume that $\bar{x}=0$. Choose one of our nice neighbourhoods $U_{c}$ for the gradient flow of $-1 / 2\left\|P_{2} x\right\|^{2}+g\left(P_{0} x\right)$ on $\left\{x: P_{1} x=0\right\}$. As in the proof of Theorem 1, we assume that $U_{\epsilon} \subseteq A$. We can now complete the proof as before. Next suppose that $\bar{x}=0$ is a non-degenerate critical point with $R\left(P_{2}\right)$ infinite-dimensional. In this case, we can take $U_{\varepsilon}$ to be a ball in $\left\{x: P_{1} x=0\right\}$ and $N_{\varepsilon}$ its boundary. Now $A \cap U_{\varepsilon}$ is compact and hence there exists $x_{0} \in\left(\operatorname{int} U_{\varepsilon}\right) \backslash A$. (Remember that $U_{\varepsilon}$ is not compact.) It is easy to construct a homeomorphism $R: U_{\epsilon} \rightarrow U_{\epsilon}$ such that $R=I d$ on $\partial U_{\epsilon}$ and $R\left(x_{0}\right)=0$. We extend $R$ to be the identity outside $U_{\varepsilon}$. Thus we can replace $A$ by a homeomorphic set $\widetilde{A}=R(A)$ such that $0 \notin \widetilde{A}$ and $f(x) \leqslant f(0)$ if $x \in \widetilde{A}$ and $x$ is close to zero. We can now deform along the gradient flow of $-(1 / 2)\left\|P_{2} x\right\|^{2}$ to obtain $A \in \mathcal{A}$ such that $f(x)<f(0)$ when $x$ is close to zero. We can now complete the proof as before. We can use the same approximation argument as in the previous paragraph to handle the case where $R\left(P_{2}\right)$ is infinite-dimensional and $\bar{x}$ is degenerate. This completes the proof of the infinite-dimensional version of Theorem 1 . Note that this improves Theorems 2.2 and 2.5 in [13] because we show that there must be a critical point with finite Morse index.

\section{REMOVABILITY OF CRITICAL POINTS}

Assume $f: \mathbf{R}^{n} \rightarrow \mathbf{R}$ is $C^{2}$ and zero is an isolated critical point of $f$. We prove a conjecture in [6] on when we can find a $C^{2}$ functional $g$ which agrees with $f$ except near zero but has no small critical points. This gives a tidier result and one which is much easier to apply than the one in [6]. In fact, as we sketch briefly, our ideas can be used to obtain a necessary and sufficient condition for removability.

Theorem 3. Assume that $n \geqslant 6, f: \mathbf{R}^{n} \rightarrow \mathbf{R}$ is $C^{2}$, zero is an isolated critical 
point of $f$, zero has trivial homotopy index and $f^{\prime \prime}(0)$ has at least one positive and one negative eigenvalue. Then there is a neighbourhood $U$ of zero and a $C^{2}$ functional $g$ which is $C^{0}$ close to $f$, which equals $f$ outside $U$ and which has no critical points in $\bar{U}$.

Remarks. The necessity of many of the conditions is discussed in [6, Section 4]. We still do not know if $g$ can be chosen $C^{1}$ close to $f$. (As pointed out in [6], it cannot always be chosen $C^{2}$ close to $f$.)

Proof: The proof is essentially the same as the proof of the proposition in [6, Section 4] except that we use the $s$-cobordism theorem (as in Kirby and Siebenmann $[10$, p.4], or Mazur [17]) instead of the $h$-cobordism theorem. As in [6], if we can prove the result with no condition on $g$, we can easily modify the proof to get $g C^{0}$ close to $f$. We choose one of our nice "box-like" neighbourhoods $U_{e}$ of zero such that zero is the only critical point of $f$ in $\bar{U}_{e}$. As in [6], we can approximate $f$ by $f_{1}$, where $f_{1}=f$ near $\partial U_{e}$ and $f_{1}$ has only non-degenerate critical points in $U_{e}$ and which are close to zero. In [6], we then used King's [9] variant of the $h$-cobordism theorem to obtain $g$ such that $g=f_{1}$ near $\partial U_{\epsilon}$ and $g$ has no critical points in $U_{\epsilon}$. Since we do not know that $U_{\varepsilon}, N_{e}, \widetilde{N}_{\epsilon}$ are simply connected this argument fails. Here we use the notation of [5]. However, if the natural inclusions $i_{1}: N_{\varepsilon} \rightarrow U_{\varepsilon}$ and $i_{2}: \widetilde{N}_{\varepsilon} \rightarrow U_{\varepsilon}$ are both simple homotopy equivalences in the sense of Cohen [4, Section 22], then we can apply the $s$-cobordism theorem. Here, as in $[\boldsymbol{6}]$, we need to check through the proof of the $s$-cobordism theorem and check that all the perturbations in $f$ can be done by purely local changes. This essentially the same as the corresponding check in [6]. The remarks in $[10$, p.4] are helpful here.

Hence the proof reduces to showing that $i_{1}$ is a simple homotopy equivalence. (A similar argument works for $i_{2}$.) Now we need four properties of simple homotopy equivalences. Firstly whether a map is a simple homotopy equivalence depends only on its homotopy class. Secondly, a homeomorphism is a simple homotopy equivalence. Thirdly if any two of $g, f, g \circ f$ are simple homotopy equivalences then so is the third (where the maps are such that $g \circ f$ makes sense). These results can be found in Cohen [4, Section 22 and appendix]. Fourthly, if $f: X \rightarrow Y$ is onto and cell-like (that is $f^{-1}(y)$ has the shape of a point (in the sense of Mardesic and Segal [14]) for $y \in Y$ and if $X$ and $Y$ are compact $A N R$ 's) then $f$ is a simple homotopy equivalence. This is the theorem in Section 4 of Lacher [12].

Now the natural inclusion $\bar{i}$ of $U_{\varepsilon}^{\prime}=U_{\epsilon} \cap f^{0}$ into $U_{\varepsilon}$ is a simple homotopy equivalence (where $\left.f^{0}=f^{-1}((-\infty, 0])\right)$. To see this, we will prove that the map $P$ of $U_{\varepsilon}$ into $U_{c}^{\prime}$ defined by following the flow till we first hit $f^{0}$ (as in $[5$, p.11]) is a simple homotopy equivalence. Since $P \circ \vec{i}$ is homotopic to the identity map and is thus a simple homotopy equivalence, it will then follow from our third statement in the previous 
paragraph that $i$ is a simple homotopy equivalence. Now we only have to prove that $P$ is cell like. Now the fibres of $P$ are points or homeomorphs of an interval except for $P^{-1}(0)$. Now $P^{-1}(0)$ is $\widetilde{I}_{\varepsilon} \cup\{0\}$ (with the notation of $[5, p .6]$ ). This is easily seen to be homeomorphic to a cone $C I_{\varepsilon}$ over $I_{\epsilon}$. Since $C I_{\varepsilon}$ is compact and contractible, it has trivial shape and hence the result follows.

By the result of the previous paragraph, it suffices to prove that the natural inclusion $i_{1}^{\prime}$ of $N_{\varepsilon}$ into $U_{\varepsilon}^{\prime}$ is a simple homotopy equivalence. Now it is proved in [5, p.11-12] that the pair $\left(U_{\varepsilon}^{\prime}, N_{\varepsilon}\right)$ is homeomorphic to $\left(\left(N_{\varepsilon} \times[0,1]\right) /\left(E_{\varepsilon} \times\{1\}\right), N_{\varepsilon} \times\{0\}\right)$. Thus it suffices to prove that the natural inclusion $\mu$ of $N_{e} \times\{0\}$ into $\left(N_{e} \times[0,1]\right) /\left(E_{e} \times\{1\}\right)$ is a simple homotopy equivalence. Now $\mu=\tau \circ i$ where $i$ is the natural inclusion of $N_{\varepsilon} \times\{0\}$ into $N_{\varepsilon} \times[0,1]$ and $\tau$ is the quotient map of $N_{\varepsilon} \times[0,1]$ into $\left(N_{\varepsilon} \times[0,1]\right) /\left(E_{\varepsilon} \times\{1\}\right)$. We can prove $i$ is a simple homotopy equivalence by a similar argument to the one we used for $\bar{i}$. Thus it suffices to prove that $\tau$ is a simple homotopy equivalence. This follows if $\tau$ is cell-like and hence if $E_{\varepsilon} \times\{1\}$ has trivial shape. We can use the proof of Lemma 2 in $[6]$ to show that $E_{\varepsilon}$ is 1 -shape connected. Now $E_{\varepsilon}$ has trivial Čech cohomology. (This follows from equation (1) of [5]). It then follows as in the proof of Lemma 2 in $[\theta]$ that $E_{\varepsilon}$ has the shape of a polyhedron. By the argument in the proof of Theorem 3 in $[\boldsymbol{\theta}]$, all the pro-groups $\pi_{j}\left(E_{\varepsilon}\right)$ are trivial and hence, since $E_{\varepsilon}$ is finite-dimensional, $E_{\varepsilon}$ has trivial shape by Theorem II.5.7. in [14]. This completes the proof.

Remarks. We could replace the assumption on the eigenvalues of $f^{\prime \prime}(0)$ by the assumption that $\operatorname{dim} N\left(f^{\prime \prime}(0)\right) \leqslant 3$. We could replace our conditions on the homotopy index and the spectrum of $f^{\prime \prime}(0)$ by the two conditions that $E_{\varepsilon}$ and $I_{e}$ have trivial shape. In this form it can be proved that the converse of Theorem 3 is true (for $n \geqslant 6$ ). The idea is that the removability assumption implies that $U_{\varepsilon}$ and $N_{\varepsilon}$ are homotopy equivalent. Thus, if $n \geqslant 6$, we obtain a necessary and sufficient condition for removability. However, it is usually not such an easy condition to check. Note that if $\nabla f$ has trivial homotopy index at zero then this condition is satisfied if and only if $E_{\varepsilon}$ and $I_{\varepsilon}$ are 1-shape connected.

\section{REFERENCES}

[1] A. Bahri and P.L. Lions, 'Remarques sur la theorie variationelle des points critiques et applications', C.R. Acad. Sci. Paris 301 (1985), 145-147.

[2] C. Bessaga and A. Pelczynski, Selected topics in infinite-dimensional topology (Polish Scientific Publishers, Warszowa, 1975).

[3] D. Clark, 'A variant of Lyusternick-Schnirelmann theory', Indiana Univ. Math. J. 22 (1973), 65-74.

[4] M. Cohen, A course in simple homotopy theory (Springer-Verlag, 1973).

[5] E.N. Dancer, 'Degenerate critical points, homotopy indices and Morse inequalities', J. Reine Ang. Math. 350 (1984), 1-22. 
[6] E.N. Dancer, 'Degenerate critical points, homotopy indices and Morse inequalities II', J. Reine Ang. Math. 382 (1987), 145-164.

[T] D. Gromoll and W. Meyer, 'On differentiable functions with isolated critical points', Topology 8 (1969), 361-369.

[8] S. Hu, Homotopy theory (Academic Press, 1959).

[9] H. King, 'The number of critical points in Morse approximation', Compositio Math. 34 (1977), 285-288.

[10] R. Kirby and L. Siebenmann, Foundational essays on topological manifolds, smoothings and triangulations: Ann. of Math. Studia 88 (Princeton University Press, 1977).

[11] J. Krasinkiewicz, 'Continuous images of continua and 1-movability', Fund. Math. 98 (1978), 141-164.

[12] R. Lacher, 'Cell-like mappings and their applications', Bull Amer. Math. Soc. 83 (1977), 495-552.

[13] A. Lazer and S. Solimini, 'Non-trivial solutions of operator equations and Morse indices of critical points of minimax type', Nonlinear Anal. 12 (1988), 761-775.

[14] I. Mardesic and J. Segal, Shape theory (North Holland, 1982).

[15] A. Marino and G. Prodi, 'Metodi perturbativi nella teoria di Morse', Boll. Un. Mat. Ital. 11 (1975), 1-32.

[16] J. Mawhin and M. Willem, 'On the generalized Morse lemma', Bull. Soc. Math. Belg. 37 (1985), 23-29.

[17] B. Mazur, 'Relative neighbourhoods and the theorems of Smale', Ann. of Math. T7 (1963), 232-249.

[18] P.H. Rabinowitz, 'Some minimax theorems and applications to nonlinear partial differential equations', 161-177, in Nonlinear Analysis, ed. L. Cesari, R. Kannan and H. Weinberger (Academic Press, 1978).

[19] P.H. Rabinowitz, 'Some aspects of nonlinear eigenvalue problems', Rocky Mountain J. Math. 3 (1973), 161-202.

[20] S. Solimini, 'On the solvability of some elliptic partial differential equations with linear part at resonance', J. Math. Anal. Appl. 117 (1986), 138-152.

[21] E. Spanier, Algebraic topology (McGraw Hill, 1966).

Department of Mathematics, Statistics and Computing Science

University of New England

Armidale NSW 2351

Australia 